\title{
Information Sharing System of Ad Hoc Networks: Based on Data Access Service
}

\author{
$\mathrm{Bo} \mathrm{Su}{ }^{*}$ \\ Xi'an Electronic Engineering Research Institute \\ Xi'an, China \\ E-mail: subwurlin@hotmail.com \\ Junhan Yang \\ Xi' an University of Science and Technology \\ Xi'an, China \\ E-mail: yang_junhan@163.com
}

\author{
Bin Wang \\ Xi' an University of Science and Technology \\ Xi'an, China \\ E-mail: 46764479@qq.com
}

\author{
Hao Wu \\ Xi'an Electronic Engineering Research Institute \\ Xi'an, China \\ E-mail: wuhao_kelly@163.com
}

\begin{abstract}
Based on high speed Ad hoc networks, researchers build a kind of information sharing system using data access service for a variety of operating system and different types of hardware source. Information sharing system using service-oriented software architecture that supports localization of operating systems and databases as well as tactical network environment, a variety of types of operating systems and hardware platforms can provide good compatibility, provides a unified secondary development interface, through future optimize and improve the network interface and load balancing control module can effectively improve the efficiency of data access, increase the number of users, improve access frequency, enhance the user experience. The results show that the new information system operates stably, smoothly and accurately. The systems deploy file servers for all of the users online fast and efficiently.
\end{abstract}

Keywords-Index Terms-Information Sharing System; Data Distribution Service; Ad Hoc Networks.

\section{INTRODUCTION}

In the future, information sharing system using a variety of types of database systems is playing an increasingly important role. For a variety of operating systems, different types of hardware platform and internet sources, how to access and use information quickly and efficiently, and establish a good information fusion sharing mechanism through analyzing information resources, is a problem to be solved urgently. Based on high-speed information sharing network and data transmission middleware, it is necessary to design a set of information sharing systems with practical and efficient information distribution, which achieves quick information access, dissemination and sharing.

Data access services ${ }^{[1-9]}$ for the application to provide unified interface of data access and storage, shield the differences of database systems and data model change, which implement logical session independence, increase developer coding efficiency and enhance reliability and portability of the system. Data access services based on standardized description way and data storage policy stored data in the network user's storage resources. Data access services supported by the type of memory database improve access speed of frequent access data. Data access services provide data synchronization according to synchronization strategy in order to complete the data synchronization of different network users and provide effective technical support means for data sharing.

\section{DATA Distribution SERVICE}

Based on the diverse computer application platform, the application software should be transplanted between different platforms. A platform needs to support and manage a variety of application software. Between hardware/software platforms and application systems, reliable and efficient data transfer or data conversion is needed, so that the collaborative capability of the different systems obtains a guarantee. In order to solve the above problems, in the process of development of computer network technology, the concept of middleware appears. From the perspective of network applications, middleware between the transport layer and application layer, exists as a software entity objectively.

In the software development process, middleware simplifies applications development of single-use platform, and masks the complexity of the underlying services for multiple platforms to simplify development and management of upper layer application services. So middleware can be defined as a software system built on the software and hardware platform with the upper level application software support.

\section{THE FRAME DESIGN OF INFORMATION SHARING SYSTEM}

Single equipment information sharing system consists of two parts: hardware and software entities. Hardware entity largely is shared information network devices such as LTE communications network equipment, high-speed networking equipment, high-speed multi-type sensor network equipment, high-speed networking radio and multi-type gateway equipment. Software entities include data transmission middleware, web service, multi-type databases, graphical interface, geographic information 
presentation system and network management software. The overall composition framework of information sharing systems based on data access middleware, and the multi-client physical model are as follows.

After various types of equipment joined information-sharing network, through the pre-configured subscription feature, it can real-time access to all types of information resources other devices shared on the network; not pre-subscription, it also can apply for subscription access lately. For intelligence resources, information resources, situation resources that various types of equipment does not require to read, subscription access can be canceled. Looking for service when the server running in a service environment attacked and can't be work, the system will find the equipment with the corresponding service functions in the network and open the service on this device via the instructions for users apply to ensure that the entire service operating environment works normally. The benefits of this design is that when some nodes of the network suffer from physical attacks, or service can't enabled in case of self-failure, service operating environment will quickly return to normal working condition.

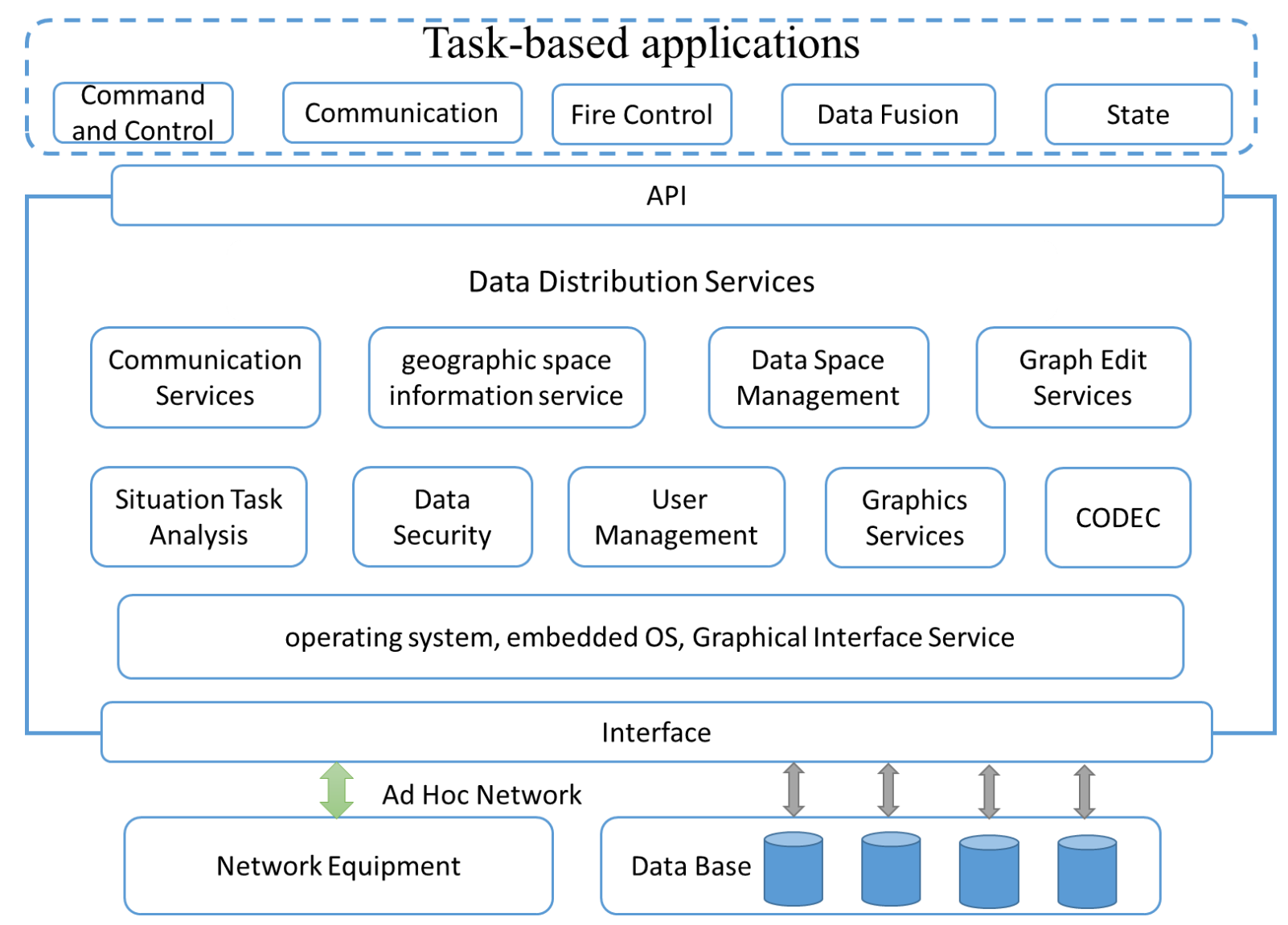

Fig. 1. The composition of information sharing system frame diagram

\section{INFORMATION SHARING SYSTEM}

\section{A. System Model}

Data access middleware by the network communication interfaces and logical session script interpreter interface, logic session handling, load balancing and database interface control module, the structure shown in Figure 2. As shown in network communication interface by the network user communication interface and server communications interfaces, it consists of two parts, which provides a communication platform for data access middleware underlying communication. The dotted line part of that is the data access middleware.

\section{B. Module Functional Description}

(i) Network Communication Interface
Network communication interfaces typically implement user logic functions session request and receive data sent to the middleware. Network communication interface with Socket programming language is between Socket and Socket with TCP/IP connection. While the network user registration information sent to the middleware, but also by the data access module. Network communication interface includes a network interface and a user communication server communication interface. Network communication interfaces typically have the ability to encrypt transmission security, and the use of process pool and multi-threading technology to improve network data transmission efficiency.

(ii) logical session processing module

Logical session processing module is responsible for implementing the task queue management, and network user authentication bidirectional forwarding logical session, 
the network user registration and data request queue scheduling management. Logical session processing module is an important part of middleware, its design will directly related to the performance and reliability of the information sharing system. It mainly deals with receiving logic session request, queuing, forwarding and so on. The workflow is to first network user registration, certification of its identity, when receiving the data network user request, the request into the request queue logical session, the session logic processing thread request queue extracted from the logical session request, according to the logic the session request to obtain the corresponding logical coding session script, and according to the logic defined by the session script logic session, issue SQL script for data access requests to the database server. The database server receives the response data sent into the result returned to the queue, and finally by sending task thread reply data to the network communication interface.

(iii) The logical session script interpreter Interface

Logical session script interpreter interface is responsible for extracting the corresponding logical conversation SQL statements from the logical session definition package. Logical session developers will logical session SQL language incorporated into the custom script language, while scripting language pack packaged into a logical conversation. When the data access middleware logic network users received session request code and the corresponding parameter data, logical session script interpreter interface via the logical session request encoding, look for the corresponding session script logic logical session definition package, lexical analysis and after syntax analysis, and depending on the circumstances add the parameter data, and ultimately form a data access request, the session task execution logic to the database server.

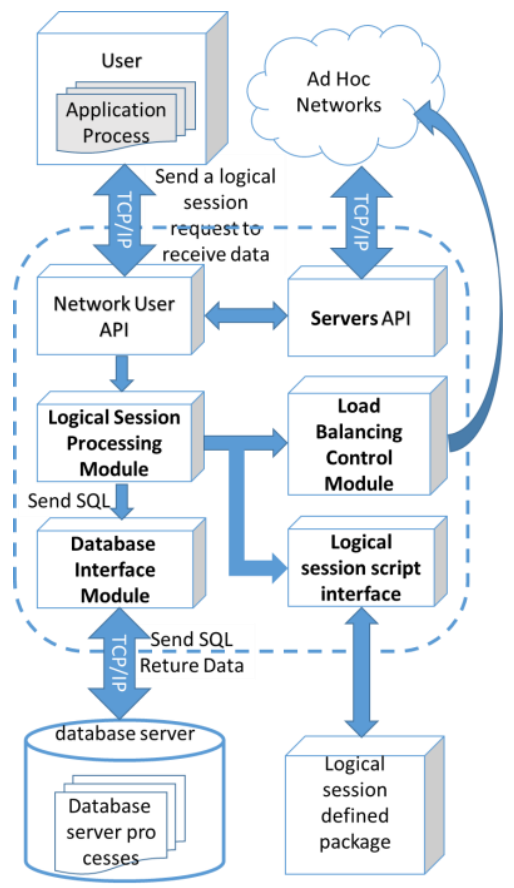

Fig 2. The data distribution services diagram (iv) The database access module

Database access module $e^{[10-11]}$ is responsible for implementing the data access middleware process, establish a link to the database, send SQL requests and return data, and other functions. To make the data access middleware to access gold positions, of dreams and other China-made large-scale databases. Since the design-time package data access interface, providing support for OCI, CLI, DBLIB, OCI, CLI, ODBC, JDBC, OLEDC, NDP, PERL, PHP, ESQL interfaces, thereby providing a cross-platform and portability database access common interface.

\section{(v) Load Balancing}

Load balancing load condition detected in real time middleware server control module, according to the load balancing algorithm, the network users request the case back to the server queue.

\section{System features}

Information sharing system using the three-tier structure of the client / server model, namely the user interface layer, session layer logic and data management. The layers are defined as follows:

(i) The user interface layer

The main function of the user interface layer is to present a visual interface to the user, easy to enter data and extract data. At the same user interface for data access provided certain security mechanisms to ensure that the specific authority level user access to data when different identities.

(ii) Logical session layer

Logical session layer between the user interface layer and data management responds to the user interface layer user requests, tasks; under the management of extracting data from the data, and outputs the data to the user interface layer.

(iii) Data management

Data management is the main function is to ensure the integrity of data storage and security, on the response to the request and data access logic session layer, which are usually achieved by gold positions, of dreams and other large domestic database server.

In this three-tier client / server mode, the logical session layer as an intermediate layer is independent of the user interface layer and data management. User and data processing logic independent sessions are conducted to ensure the unity and integrity of the data.

The main features of the distributed system as follows: (i)It provides a simple user interface to achieve import and export data, (ii)It provides a logical conversation function as links between users and data, (iii)providing distributed management capabilities, (iv)It provides a safety mechanism and load balancing mechanism with multiple hardware platforms and different operating systems support for distributed applications and services, data management, logical session layer as an intermediate layer to protect the security of data, (v)It provides $24 \times 7$ hours of data access high reliability and stability, and the system is easy to maintain and upgrade when the business logic of 
the session layer of middleware change. The system response rapidly and is easy to expand.

\section{V.TEST AND VERIFICATION ENVIRONMENT}

Test environment using ad hoc network environment of multiple network terminal equipment. The operating system use the Keylin Linux with a kernel version 3.10.27 or reworks, one of the network terminal emulation command post, which installing a service-oriented operating environment and development graphic display terminal integrated display control interface. The other network terminal emulation reconnaissance unit, install surveillance data source and service-oriented simulation generates operating environment. When testing, the generator generates reconnaissance data from multiple reconnaissance units and sharing reconnaissance information to the network. Command post receives reconnaissance information sharing by subscription. It is achieved a real-time target trajectory displayed on the map, as shown Fig. 3. After 500 hours test to verify the accuracy and reliability of the information sharing system, the result show that it is stable and practical.

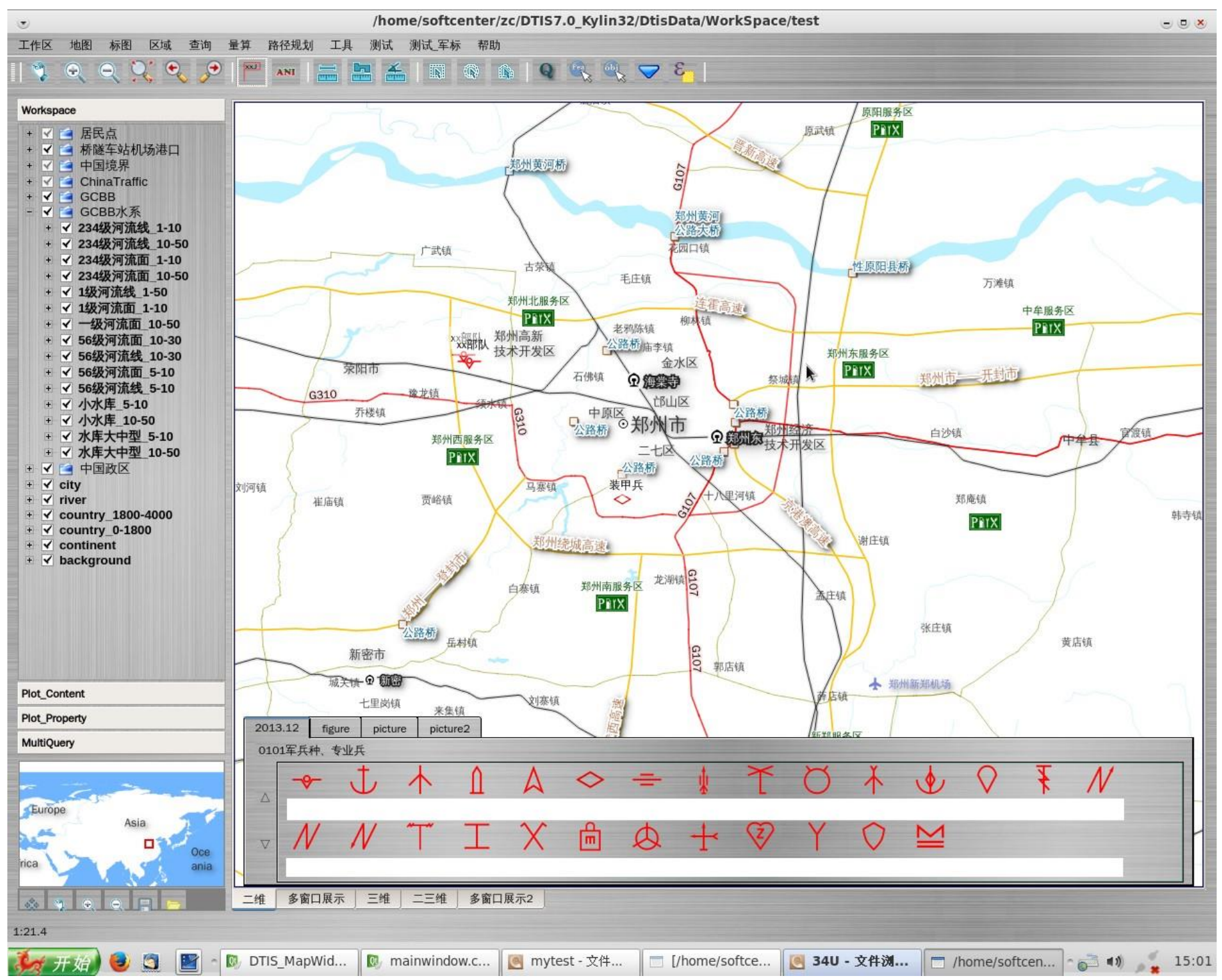

Fig 3. Real-time information sharing interface diagram

\section{APPLICATION AND PROSPECT}

Information sharing system using service-oriented software architecture that supports localization of operating systems, databases as well as tactical network environment, a variety of types of operating systems and hardware platforms can provide good compatibility and a unified secondary development interface. In the future, through optimizing and improving the network interface and loading balancing control module, it can effectively improve the efficiency of data access, increase the number of users, improve access frequency, and enhance the user experience.

\section{ACKNOWLEDGMENT}

The authors are grateful to the support of Scientific Research Program Project of Education Department of Shaanxi Province (No. 14JK1487), Scientific Research 
Program Project of Xi'an Beilin District Science and Technology Bureau (No. GX1512) and Cultivating Fund of Xi'an University of Science and Technology Research (No. 2014075).

\section{REFERENCES}

[1] P. C. Gerardo. OMG Data Distribution Service: Architectural Update.2004 IEEE Military Communications Conference, 2004, 2:961 967.

[2] G. P. Castellote. DDS Spec Outfits Publish Subscribe Technology for the GIG. COTS Journal, 2005.

[3] F. F. Li, X. Z. Yu, G. Wu. Design and Implementation of High Availability Distributed System Based on Multi-Level Heartbeat Protocol. IITA International Conference on Control, Automation and Systems Engineering. IEEE, 2009:83 87.

[4] G.L. Feng, Q. F. Gu. Design and Implementation of a Real-Time Middleware Based on DDS.3rd International Conference on Information, Electronic and Computer Science, 2011.

[5] Fiore, Sandro, et al. Advances in the GRelC Data Access Service. Parallel \& Distributed Processing with Applications .ispa.international Symposium (2008):849-854.
[6] Liao, Chien-kai, Li, Yu-hua, Lai, Kuang-fu, et al. System for providing a bidirectional data access service and method thereof: US, US20130179492 A1[P]. 2014.

[7] Plante, Raymond, et al. "IVOA Recommendation: SimpleDALRegExt: Describing Simple Data Access Services." Eprint Arxiv (2014).

[8] Li Hui;Sun Wenhai;Li Fenghua;Wang Boyang;State Key Laboratory of Integrated Services Networks(Xidian University);Institute of Information Engineering, Sciences; C A O. Secure and Privacy-Preserving Data Storage Service in Public Cloud[J]. Journal of Computer Research \& Development, 2014, 51(7):1397-1409.

[9] T.Ma, J. Hillston, S. Anderson. Evaluation of the QoS of Crash-Recovery Failure Detection. In SAC' 07: Proceedings of the ACM Symposium on Applied Computing, 2007:538 542.

[10] Z. H. Hou, Y. X. Huang, S. Q. Zheng, et al. Design and Implementation of Heartbeat in Multi-Machine Environment. Advanced Information Networking and Applications. ANIA2003. 17th International Conference, 2003:583 586.

[11] S. D. Huston, J. C. E. Johnson, U. Syyid. Practical Design Patterns for Network and Systems Programming. Beijing: Publishing House of Infopower, 2004: 5 155. 